\title{
Some challenging aspects of ductile materials
}

\author{
P. Brož \\ Professional Association for Science, Research and Consultancy, \\ Czech Republic
}

\begin{abstract}
Crack tip blunting due to tension loads and re-sharpening of the tip in the course of unloading are two fundamental fatigue crack propagation processes in yielding metals. Using an elastic perfectly plastic material simulation, crack propagation analysis was performed up to several hundreds of complete cycles by means of remeshing at some phases of plastic deformation, including computations of the influence of overloads and compressive underloads. For the first several cycles, seaming was indicated, mainly when the crack surface was compressed, resulting in something that seems to suggest striations. Plastic cycling naturally renders a fair amount of crack growth per cycle, crack closure at the tip is predicted in each cycle next to an initial transient phase, and an overload ends in a reduced increase in crack growth.

After introducing a single overload, tests on fatigue crack growth were performed with specimens of austenitic steel. The variables are $K_{\max }$ of the overload and the thickness of the specimen. The latter factor is of crucial importance when considering plane strain/plane stress. For this investigation, the following methodologies were employed: (i) fractographic observations, (ii) deformation analysis, and (iii) crack growth records.

For modelling ductile fractures in structural materials, applying mechanism- based conceptions, two numerical methods can be used for simulating the material failure process. Firstly, voids are unambiguously regarded and simulated applying refined finite elements. Secondly, the material comprising the voids is regarded as a homogenized continuum described by porous plasticity simulations.

Keywords: crack stopping, ductile crack growth, peak load, remeshing, single overloads, striations, surface folding, transient stage, void growth and coalescence.
\end{abstract}




\section{Introduction}

Finite strain plasticity to simulate the large deformations round the crack-tip, when analyzing crack blunting, was used in $[1,2]$. At the same time, the first two or three full load cycles could be studied owing to very sturdy mesh distortion outside the tip. It was learned that the crack-tip opening displacement demonstrates transient characteristics, such that for many cycles at first there is no crack closure at the tip when the minimum load is achieved in each cycle but from that time the growth pattern forms in relation to a steady-state when closure occurs in each cycle. It is desirable to treat the mesh sensitivity of the crack propagation predictions by crack-tip blunting and re-sharpening. These subjects have been set up in detail by Tvergaard in his latest treatise [3]. Levkovitch et al. in [4] employed remeshing procedures for a material characterized by crystal plasticity. The interesting disparity is that they derived surface folding subject to compression, resulting in something that resembles induction of striation.

\section{Study approach}

Let us consider a plate weakened by a center crack subject to a load of intensity $p$, at the transverse edges (fig. 1). The solution unwittingly means the fact that the mode I singularity field produces round the crack-tip if the crack opens whereas when the crack closes under compressive loading, the state of stress changes to uniaxial compression under circumstances of plane strain. The plate possesses load-free sides. The region $-a \leq x \leq w-a$ and $0 \leq y<h$ lengthwise being one quarter of the plate is analyzed numerically. The crack surface $-a<x^{1}$ $<0$ is traction free, $T^{1}=T^{2}=0$ if the crack is open (on the surface it holds: $u^{2}>$ $0)$. Anyhow, when contact is present at a point of the crack surface, $\dot{u}^{2}<0$ in the case: $u^{2}=0$, these requirements are substituted by the contact condition that the displacement normal to the crack surface equals zero, $u^{2}=0$, and the tangential traction on the crack surface is zero, $T^{1}=0$ : The tension free boundary conditions are re-taken at this point of the crack if the compressive contact force is nearly to vanish, it is $\dot{T}^{2}>0$ for $T^{2}=0$. For the cracked plate tensioned, in compliance with [5], the stress intensity factor $K_{1}$ is equal to

$$
K_{1}=c p \sqrt{\pi a}
$$

where $c \cong 1.19$ for $a / w=1$

$$
\text { or } K_{1}=\sigma \sqrt{\pi a} \cdot F_{I}(\alpha, \beta), \alpha=\frac{2 a}{W}, \beta=\frac{2 H}{W} \text { and } \frac{H}{W}=4
$$

according to fig. 1 and table 1 .

When specifying $K_{1}$, this value obtained from Eq. (1) is the matter, if the applied load $p$ is known, and like that a negative value of $K_{1}$ is an example of the respective negative value of $p$ although contact will have occurred on the crack surface and so the mode 1 singularity field is not pertinent. To calculate $K_{1}$, the initial crack length is applied since the difference consistent with employing the current crack length is irrelevant in the events contemplated. 


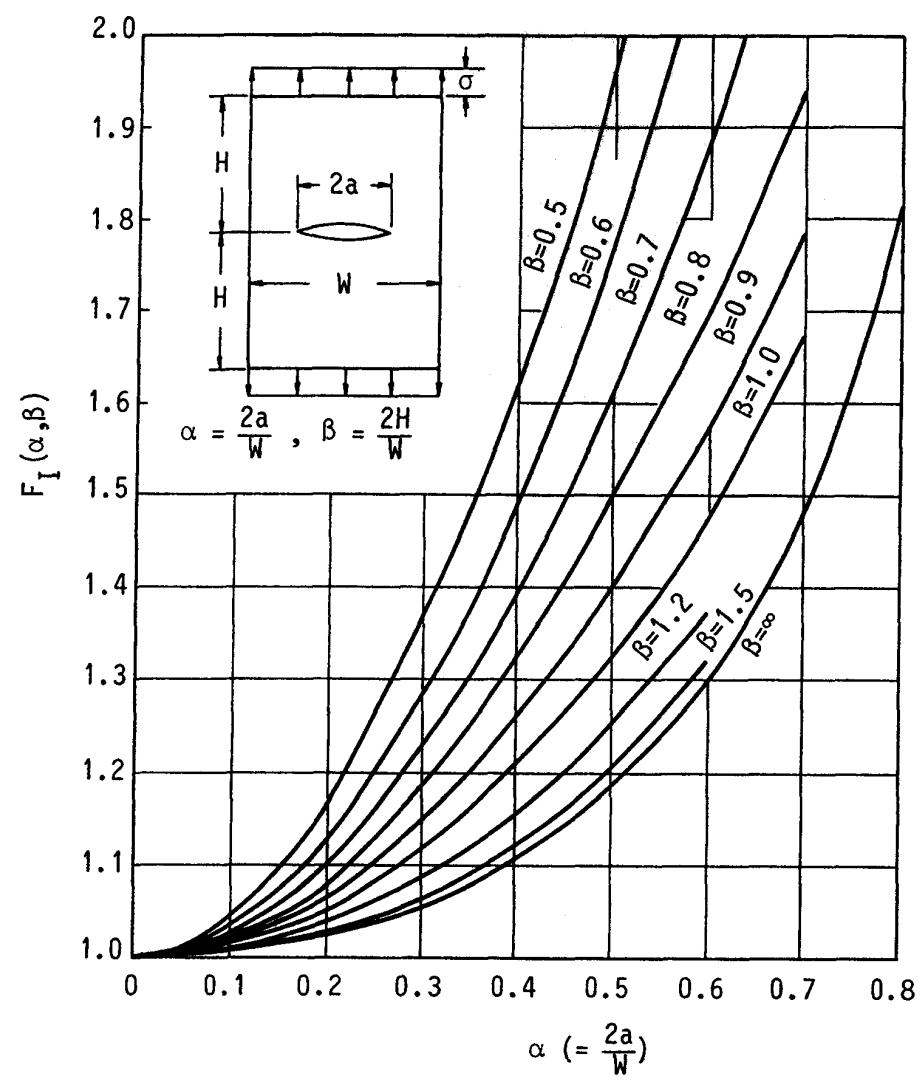

Figure 1: Boundary correction factors, $F_{\mathrm{I}}(\alpha, \beta)$.

Table 1: $\quad$ Boundary correction, $F_{\mathrm{I}}(\alpha, \beta)$, for the case of uniform tension [5].

$$
\left(F_{I}=K_{I} / \sigma \sqrt{\pi a}\right)
$$

\begin{tabular}{|c|c|c|c|c|c|c|c|c|c|c|c|}
\hline \multirow{2}{*}{$\boldsymbol{\alpha}$} & \multicolumn{10}{c|}{$\boldsymbol{\beta}$} \\
\cline { 2 - 14 } & 0.4 & 0.5 & 0.6 & 0.7 & 0.8 & 0.9 & 1.0 & 1.2 & 1.5 & 1.8 & $\infty$ \\
\hline 0 & 1.000 & 1.000 & 1.000 & 1.000 & 1.000 & 1.000 & 1.000 & 1.000 & 1.000 & 1.000 & 1.000 \\
\hline 0.1 & 1.069 & 1.046 & 1.033 & 1.026 & 1.021 & 1.017 & 1.014 & 1.010 & 1.007 & 1.006 & 1.006 \\
\hline 0.2 & 1.256 & 1.175 & 1.130 & 1.103 & 1.083 & 1.067 & 1.055 & 1.039 & 1.029 & 1.025 & 1.025 \\
\hline 0.3 & 1.520 & 1.371 & 1.285 & 1.228 & 1.184 & 1.150 & 1.123 & 1.088 & 1.066 & 1.060 & 1.058 \\
\hline 0.4 & 1.843 & 1.629 & 1.497 & 1.400 & 1.323 & 1.262 & 1.216 & 1.158 & 1.122 & 1.112 & 1.109 \\
\hline 0.5 & 2.247 & 1.967 & 1.773 & 1.619 & 1.496 & 1.403 & 1.334 & 1.251 & 1.203 & 1.190 & 1.187 \\
\hline 0.6 & 2.806 & 2.424 & 2.123 & 1.883 & 1.702 & 1.572 & 1.481 & 1.38 & 1.32 & 1.31 & 1.303 \\
\hline 0.7 & 3.67 & 3.04 & 2.55 & 2.19 & 1.94 & 1.78 & 1.68 & - & - & - & 1.488 \\
\hline
\end{tabular}


In all eventualities, the specimen is originally stress free $\left(K_{1}=0\right)$. Afterwards, the load at the edges of the sample is cycled between quantities $p_{\max }$ and $p_{\min }$, commensurable to $\left(K_{1}\right)_{\max }$ and $\left(K_{1}\right)_{\min }$. A reference value $K_{0}$ of the stress intensity factor is applied to put on the issues, and a reference dimension of the plastic region for $K_{1}=K_{0}$ is determined being

$$
R_{0}=\frac{1}{3 \pi}\left(\frac{K_{0}}{\sigma_{Y}}\right)^{2}
$$

The material is characterized by non-hardening perfect plasticity for that the hysteresis loop in not erased by any number of cycles. $J_{2}$ flow theory with isotropic hardening would not be well fitted to symbolize the hysteresis loops, as a sufficient quantity of hardening will fully put plastic yield in the course of cycling down. But still, good conformity with experiments for a hardening material can be obtained by applying special cyclic plasticity simulations.

Finite strains are presented in the computations when using coordinates in a Lagrangian formulation of the field equations in which $g_{\mathrm{ij}}$ and $G_{\mathrm{ij}}$ stand for metric tensors in the reference configuration and the current configuration, respectively, with determinants $g$ and $\mathrm{G}$, and $\eta_{\mathrm{ij}}=1 / 2\left(G_{\mathrm{ij}}-g_{\mathrm{ij}}\right)$ is the Lagrangian strain tensor. The contravariant components $\tau^{\mathrm{ij}}$ of the Kirchhoff stress tensor on the prevalent base vectors are expressed to the components of the Cauchy stress tensor $\sigma^{\mathrm{ij}}$ by the relation $\tau^{i j}=\sqrt{G / g} \sigma^{i j}$. Later, in the finite strain formulation for a perfectly plastic material with the Mises yield surface, the incremental stress-strain relationship has the following form, $\tau^{\mathrm{ij}}=L^{\mathrm{ijkl}} \eta_{\mathrm{kl}}$. The instantaneous moduli are declared as functions: of Young's modulus $E$, Poisson's ratio $v$, and the yield stress $\sigma_{\mathrm{f}}$. The material parameters employed are $\sigma_{\mathrm{f}} / E=0.003$ and $v=$ $1 / 3$. The linear finite element method used is based on an incremental variant of the principle of virtual work, when introducing the displacement fields approximated by dint of plane strain 8 - noted isoparametric elements. A uniform $60 \times 5$ mesh region near the crack-tip of the total length $l$ has the length $1 / 4$ ahead of the crack-tip and the length $3 l / 4$ behind the crack-tip. A reference length is specified as $l_{0}=0.00075 a$. The remeshing technique represents a plane strain variant of a procedure first brought in an analogous axisymmetric program by Pedersen [6]. A reference value $K_{0}$ for the stress intensity factor $K_{1}$ in mode I, is selected like this that the corresponding quantity of the J-integral, $J_{0}=K_{0}^{2}(1$ $\left.v^{2}\right) / E$, fulfils $J_{0} /\left(\sigma_{\mathrm{f}} l_{0}\right)=0.3951$. Afterwards it follows that the reference value (2) for the dimension of the plastic region at $K_{1}=K_{0}$ equals $R_{0}=15.72 l_{0}$. In this way, for $\left(K_{1}\right)_{\max }=K_{0}$, the reference length for the plastic region is much smaller than the crack length, $R_{0} / a=0.0118$.

\section{Predictions by simulation}

Completely cyclic loading was particularized like this that the peak values of $K_{1}$ conforming with (1) are $K_{\max }=K_{0}$ and $K_{\min }=0$, commensurable to a load ratio $R=K_{\min } / K_{\max }=0$. 
The analysis embracing 700 full cycles was repeated, for one overload $\left(K_{1}\right)_{\mathrm{ov}}$ $=1.4 K_{0}$ applied in the high peak of cycle 101 . These studies were performed for a $60 \times 5$ uniform mesh of length $l=l_{0}$ in the near-tip zone. Deformed crack-tip meshes are demonstrated in fig. 2 at the high peaks 100 and 101, and the next low peak where there is obviously no crack closure at the tip.

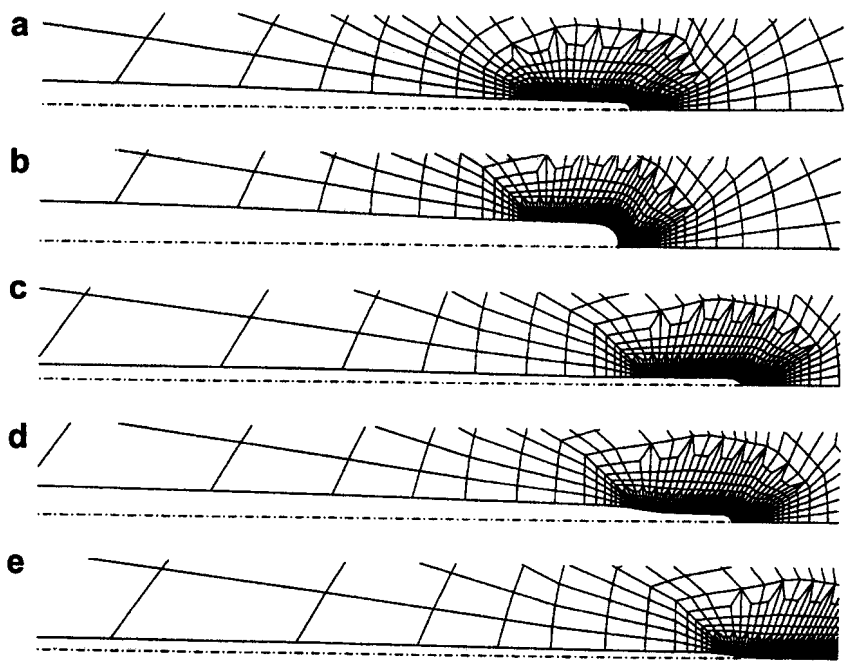

Figure 2: $\quad$ Meshes deformed for maximal loads in the case of $\left(K_{1}\right)_{\max }=K_{0}$, $\left(K_{1}\right)_{\min }=0$; (a) maximal peak No. 100, (b) maximal peak No. 101, at overload $\left(K_{1}\right)_{\mathrm{ov}}=1.4 K_{0}$, (c) minimal peak No. 101, (d) maximal peak No. 150 and (e) minimal peak No. 150. The reference length 10 is demonstrated being size-scale (from [3]).

At last, the deformed meshes at the high peak and the low peak 150 indicate that here crack closure again is present near the tip. The movement of the cracktip between the high peak and the low peak in the same cycle is owing to the reality that the mesh plots are traced with the location of the specimen center lines set in space and so elastic deformations of the specimens in the course of the cycle do the location of the crack-tip move slightly back and forth.

The predicted progress of the cyclic crack propagation $\Delta a$ against the peak number $n$ is demonstrated in fig. 3a for the completely cyclic loading and for the eventuality when an overload is applied in cycle No. 101. The number of cycles, $N$, is $N=n / 2$. The case involving the overload demonstrates undoubtedly reduced values of $\mathrm{d} a / \mathrm{d} N$ directly after the overload, and here the crack growth halted totally round $N=500$. The motion of the crack-tip between the high peak and the low peak gives rise to the somewhat large thickness of the curves in fig. 3a. The change of the crack-tip opening displacement, CTOD, in the course of the cyclic loading is demonstrated in fig $3 \mathrm{~b}$ for the instance corresponding to the overload. Due to symmetry, CTOD is twice the opening indicated in fig 2, and 
the CTOD is here measured being the largest opening within the scale of the uniform mesh zone near the tip. It was learned in [7] that there is an initial transient phase embracing no crack closure near the tip.
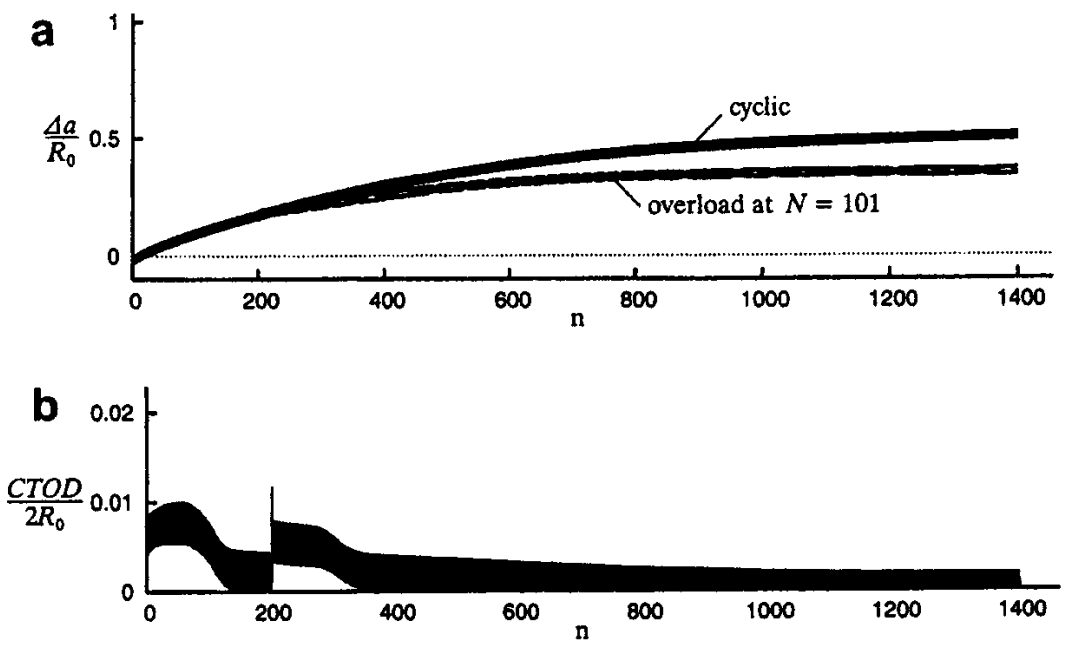

Figure 3: $\quad$ Progress of cyclic crack propagation $\Delta a$ and crack-tip opening displacement CTOD versus maximum number $n$ (where cycle number $N=n / 2)$. Cyclic loading, $\left(K_{1}\right)_{\max }=K_{0}$ and $\left(K_{1}\right)_{\min }=0 \mathrm{v}$. case for one overload, $\left(K_{1}\right)_{\mathrm{ov}}=1.4 K_{0}$ in maximal peak No. 101: (a) $\Delta a$ v. $n$ and (b) CTOD v. $n$ for the overload case (from [3]).

The overload has been applied after the termination of the mentioned phase, and it is obvious that the overload has led to a new transient stage rather analogous to that considered at the outset of cyclic loading. Undoubtedly, the numerical blunting re-sharpening simulation is competent to predict some of the aspects well known for experimental watching of fatigue crack propagation.

For a crystal plastic material, Levkovitch et al. [4] applied remeshing to give an account of the blunting and re-sharpening process during fatigue crack growth, only their predictions differ recognizably from those learned in [8]. As far back as in the first cycle, beginning from a sharp crack, the authors [3] obtained some waviness on the surface of the blunted crack point that developed into a folding of the crack surface in course of the compression portion of the cycle. Nothing but partial unfolding is present in the tensile part of the next cycle and further in the subsequent compressive part, a powerful fold evolves again.

What is left of such folds could develop into something that looks striations on the fatigue crack surface though these studies taken into account no more than the two first cycles so it is not possible to formulate distinct opinions. The authors referred to given that similar issues are obtained for isotropic plasticity. A possible reason of the difference may be that a finer mesh was employed in [4]. To analyze this point, a computation for $K_{\max }=1.4 K_{0}$ and $K_{\min }$ 
$=0$ was performed with half the element size in the near-tip zone. These calculations use a uniform mesh of length $l=3 l_{0}$, and the former computations applied a 60x5 uniform mesh whereas the new computations use a 120x8 uniform mesh. The deformed finer mesh is indicated in fig. 4 at high peak No 1 and at low peak No 1 but these deformed meshes derived by the current numerical technique demonstrate no folding of the crack surface resulting in something like striations. Nevertheless, a comparing of the two issues for the progress of the cyclic crack growth $\Delta a$ in fig. 5 shows that there is a dissimilarity, in that the finer mesh predicts a higher rate of crack growth at the onset.

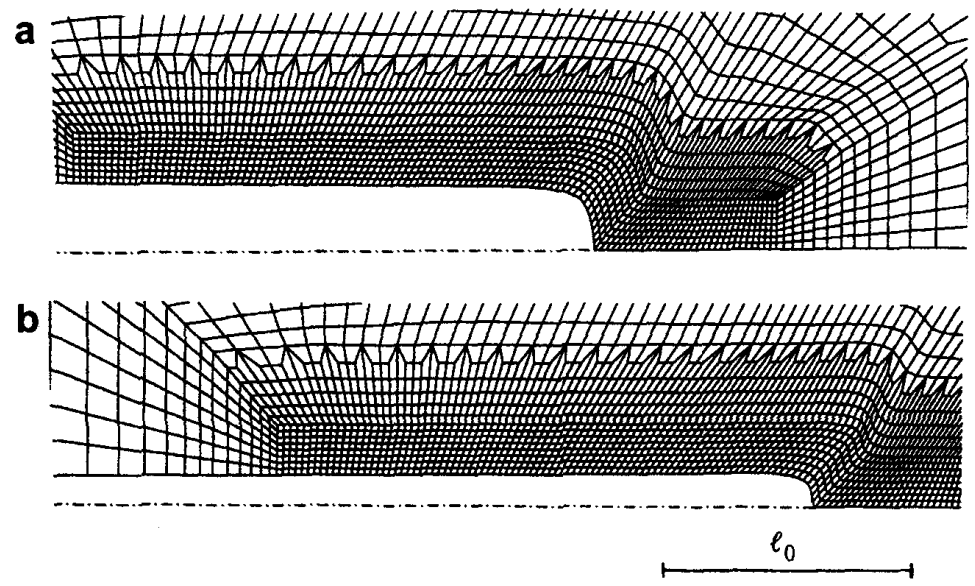

Figure 4: $\quad$ Deformed meshes at maximal loads for $\left(K_{1}\right)_{\max }=1.4 K_{0},\left(K_{1}\right)_{\min }=$ 0 , with $120 \times 8$ mesh refined, in near-tip zone: (a) maximal peak No. 1 , and (b) minimal peak No. 1.

Maybe an explanation of the difference between fig. 4 and the results of [4] consists in that the new mesh in fig. 4 was calculated automatically at each remeshing being illustrative of the smooth blunted crack surface while in [4] the new mesh was set up manually. If folding at the crack surface is extremely sensitive to geometrical imperfections this could shed light on the difference. In [4], the crack surface folding was predicted as far back as in the first and second cycles while the calculation in figs 4 and 5 was extended up to 500 total cycles without predicting any folding of the crack surfaces during the compressive portions of the cycles.

\section{Void representation and overload regime}

Further, the simulating ductile fracture in structural materials applying the mechanism - based approach is the matter, namely both the porous continuum way of tackling and the discrete void concept. Concurrently, voids are 


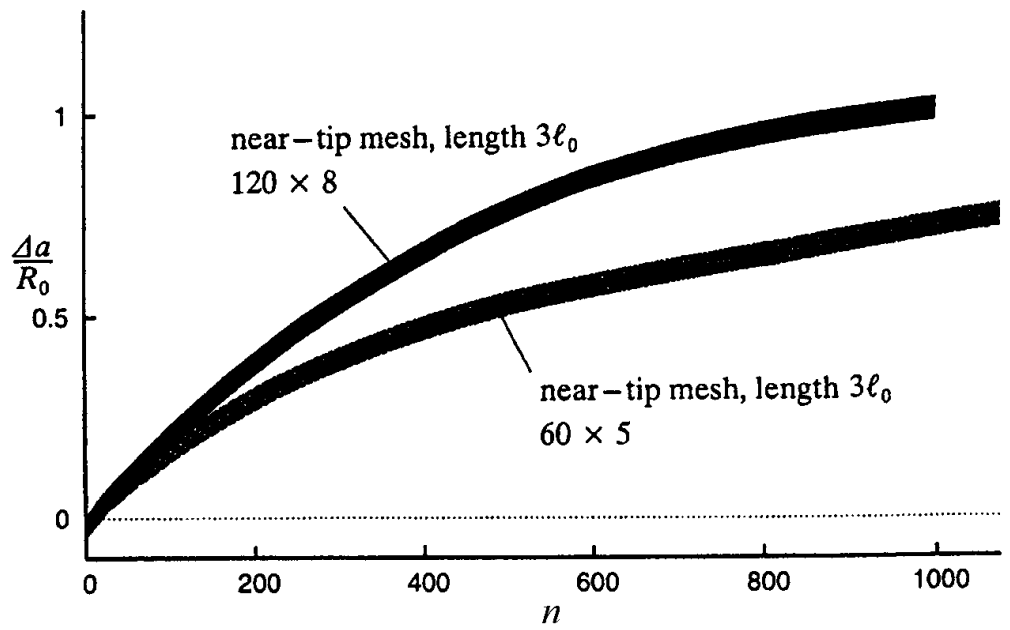

Figure 5: Assessment of cyclic crack growth $\Delta a$ v. maximum number $n$ (where cycle number $N=n / 2$ ). Cyclic loading, $\left(K_{1}\right)_{\max }=1.4 K_{0}$ and $\left(K_{1}\right)_{\min }=0$.

contemplated expressly and modelled using refined finite elements, and in the second reading, the void-including material is regarded as a homogenized continuum determined by porous plasticity simulations.

According to [9], we took into account the instance of material failure when localizating plastic flow in the inter-void ligament a we got the failure criterion being a function of the stress triaxiality ratio and the Lode angle. To that end the finite element analysis functions, viz. of the void containing a representative material volume subject to various macroscopic stress states. After employing boundary layer model with discrete voids, two void propagation mechanisms, void-by-void growth are researched into. The calibrated computational model is used to prognosticate crack extension in samples having different initial crack compositions.

In [10], the interruption by a single overload was studied, of fatigue crack propagation experiments. Variables of the testing program represented the severity of the OL and the specimen thickness, the latter factor from the standpoint of plane strain /stress implications. The outcomes corroborated the delayed retardation thing. Fractographic observations are to be made with special attention paid to crack extension in the course of the OL and base line cycles following the OL. Striation observations demonstrate an increased crack growth rate in a small number of cycles after applying the OL. That is an unexpected phenomenon, which could be made with innovative fractographic techniques. It is tied in with crack tip blunting and crack tip opening displacements. The deformation analysis and crack growth records were performed as well. The principal controlling argument for such investigation was to analyze the topology obtained during the OL and subsequent cycles, and to clarity some initial faster crack propagation. 


\section{Conclusion}

Plastic cycling exclusively yields an understanding quantity of crack growth per cycle, crack closure at the tip is predicted in each cycle following a start-up transient phase, and an overload results in a reduced crack propagation rate succeeded by crack stopping in the course of many cycles. Folding of the crack surface, which could end in striations, was predicted in the paper by Levkovitch et al in the first few cycles though it was not learned by the procedure after [3]. A mesh sensitivity was inferred in that at first the finer meshes perform a marginally higher rate of crack propagation. It is anticipated that the dissimilarity might be owing to differences in the technique used to generate new meshes, supposing that folding at the crack surface is very sensitive to geometric imperfections.

Using the small extent yielding, boundary layer simulation comprehending discrete voids stood for in the crack tip zone by refined finite element mesh, the influences of the initial void interval, void arrangement, void shape and void volume fraction fracture initiation toughness were examined. The issues can be employed to clarify the watched fracture toughness anisotropy in industrial alloys. With the help of a node-release methodology, crack propagation and fracture resistance curve may be predicted.

The plastic blunting of the crack tip when overloading gives rise to a lower crack closure level in the first few cycles after the overload, as demonstrated by larger striation interval in cycles mentioned. The early crack growth characteristics after overloading and the growth rate in the first cycles are not influenced by the state of stress.

The quicker crack propagation in a few cycles after use of an overloading suggests an unforeseen interaction effect that may be important for fracture mechanics models for the prediction of fatigue crack growth subject to variableamplitude loads.

\section{Acknowledgements}

The author gratefully acknowledges the financial support of the presented research by the Grant Agency of the Czech Republic (project No. 103/06/1382) and the Grant Agency of the Academy of Sciences of the Czech Republic (project IAA 200710604).

\section{References}

[1] Gu, I., Ritchie, R.O., On the crack-tip blunting model for fatigue crack propagation in ductile materials. In: Panoutin, T.L. et al. (Eds.), Fatigue and Fracture Mechanics: Twenty-Ninth Volume. ASTM STP 1332, 552564, 1999.

[2] Tvergaard, V., Hutchinson, J.W., Crack growth per cycle by blunting and void growth. In: Blom, A.F.(Ed.), Fatigue 2002: EMAS, UK, vol. 1/5, pp. 107-116, 2002. 
[3] Tvergaard, V., Mesh sensitivity effects on fatigue crack growth by crack-tip blunting and re-sharpening. International Journal of Solids and Structures, 44, pp. 1891-1899, 2007.

[4] Levkovitch, V., Sievert, R., Svendsen, B., Simulation of fatigue crack propagation in ductile metals by blunting and re-sharpening. In: Proceedings of ICF11, Turin, Italy (on CD), 2005.

[5] Isida, M., Effect of width and length on stress intensity factors of internally cracked plates under various boundary conditions, Int. J. Frac, 7, No. 3, pp. $301-316,1971$.

[6] Pedersen, T.O., Remeshing in analysis of large plastic deformations. Computers and Structures, 67, pp. 279-288, 1998.

[7] Tvergaard, V., On fatigue crack growth in ductile materials by cracktip blunting. Journal Mechanics Physics Solid, 52, pp. 2149-2166, 2004.

[8] Tvergaard, V., Effect of underloads or overloads in fatigue crack growth by crack-tip blunting. Engineering Fracture Mechanics, 73, pp. 869-879, 2006.

[9] Kim, J., Zhang, K., Gao, X., Modeling of ductile fracture: Application of the mechanism-based concepts. International Journal of Solids and Structures, 44, pp. 1844-1862, 2007.

[10] Bichler, Ch, Pippan, R., Effect of single overloads in ductile metals: A reconsideration. Engineering Fracture Mechanics, 74, pp. 1344 - 1359, 2007. 